CONCEPTO ABSOLUTO Y GRAMÁTICA ABSOLUTA: EL CUSANO ENTRE NEOPLATONISMO E IDEALISMO

Absolute concept and absolute grammar: the Cusano between Neoplatonism and idealism

\title{
Claudia D’Amico'
}

\begin{abstract}
RESUMEN
En este artículo analizo la noción de concepto absoluto en el pensamiento de Nicolás de Cusa. Esta noción atribuida a Dios revela la uni-trinidad entendida como reflexión, pensamiento del pensamiento o definición que se define a sí misma. Cusano concibe esto utilizando el modelo de subjetividad humana y plantea una gramática absoluta que la mente explica como actualización de sus posibilidades. De esta manera, la correspondencia entre realidad y pensamiento propia del neoplatonismo y el idealismo tiene notas singulares en la filosofía cusana.
\end{abstract}

Palabras clave: Concepto absoluto. Principio intellectual. Mente. gramática absoluta. Neoplatonismo. Idealismo.

\begin{abstract}
In this paper I analyze the notion of absolute concept in the thought of Nicolas de Cusa. This notion attributed to God reveals the uni-trinity understood as reflection, thought of thought or definition that defines itself. Cusanus conceives this by using the model of human subjectivity and poses an absolute grammar that the mind explains as actualization of its possibilities. In this way, the correspondence between reality and thought typical of Neoplatonism and idealism has singular notes in cusan philosophy.
\end{abstract}

Keywords:Absolute Concept. Intellectual Principle. Mind. absolute grammar. Neoplatonism. Idealism.

En 1972 Werner Beierwaltes publicó un libro fundacional: Platonismus und Idealismus. En la breve introducción llama la atención sobre el coordinante "und" que reúne ambas tradiciones filosóficas: en efecto no se trata de asimilar una a la otra, ni siquiera de establecer una necesaria conti-

1 Consejo Nacional de Investigaciones Científicas y Técnicas, Universidad de Buenos Aires, Universidad Nacional de La Plata. E-mail: claudiadamico@yahoo.com.ar. ORCID: https://orcid.org/0000-0003-4388-3133. 
nuidad sino de confrontar una estructura de pensamiento y establecer ciertas afinidades relativas al método y al objeto. Como sabemos, los escritos en los cuales Beierwaltes vuelve sobre esto son muchos y su visión del platonismo se vuelve más bien al neoplatonismo, sobre todo de Plotino y Proclo, en vinculación con el idealismo de Schelling o Hegel (Beierwaltes, 1980; 2002).

De hecho estos pensadores alemanes tuvieron acceso directo a los textos de los neoplatónicos tardoantiguos y en sus páginas hacen referencia a ellos. Sin embargo, el propio W. Beierwaltes ha señalado que tal vinculación no sería completamente comprensible si no se considera la mediación de los autores medievales y renacentistas como Eriúgena, el Cusano o Bruno. En su célebre Identität und Differenz. Zum Prinzip cusanischen Denkens estudia la vinculación entre Cusano y Hegel. En este caso, si bien manifiesta que Hegel no parece haber leído al Cusano, sin embargo señala algunas afinidades en dos temas que se vinculan entre sí: la comprensión del principio y el tema de la reflexión absoluta. Con posterioridad se ha investigado con profundidad una pista que ofrece el mismo Beierwaltes: Hegel pudo haber conocido la doctrina cusana a través de los textos de Historia de la Filosofía que lo mencionan (Strok, 2015; Stanfield, 2017: X-XVIII).

Sea de esto lo que fuere, la comprensión de una identidad dinámica y autoreflexiva es un punto común entre neoplatonismo e idealismo y la filosofía cusana puede ser entendida como receptora de la tradición neoplatónica pagana y cristiana, y a la vez como anunciadora de desarrollos germanos ulteriores.

Sabemos que el entramado de fuentes en las que abreva el pensamiento cusano es complejo, con todo también sabemos que los autores de la tradición neoplatónica cumplen en él un rol definitivo. Esto se vuelve muy patente en el tópico de la consideración de un principio uno que es a la vez intelecto. Las claves de esta consideración se encuentran, por una parte, en la continuidad misma del neoplatonismo post-plotiniano especialmente en el pensamiento de Proclo y en la recepción que de él hace Dionisio pseudo Areopagita; y, por otra, en la asimilación y transfiguración de las hipóstasis neoplatónicas que en el occidente latino habían sido reconvertidas en Trinidad cristiana por Mario Victorino y Agustín de Hipona. A todos estos autores que son leídos por él de manera directa debemos sumar otras líneas de 
llegada de esta tradición como el anónimo Liber de Causis y su recepción en algunos autores de la escuela de Colonia que el Cusano conoce muy bien.

En este artículo, me detendré en la noción de concepto absoluto y su vinculación con el concepto mental en la filosofía cusana a fin de poner de manifiesto de qué modo la henología cusana, de clara inspiración neoplatónica, es medida desde una filosofía de la subjetividad en la cual el simil del lenguaje humano aporta una clave de lectura que permite mostrar negativamente algo de lo absoluto. De esta manera, a la correspondencia entre ser y pensar común a ambas tradiciones filosóficas el Cusano suma una comprensión de la subjetividad humana vacía de contenidos que preludia desarrollos ulteriores de las filosofías moderna y contemporánea.

\section{Concepto absoluto, authypostaton, definición absoluta.}

Desde los fundacionales trabajos de E. Cassirer, cierta línea interpretativa ha puesto énfasis en el diálogo De Mente, el tercero de la serie Idiota (1450), para mostrar la prioridad del tema de la subjetividad en el pensamiento cusano (Benz, 1999; Zeyer, 2015). Ciertamente el Cusano subraya en esta obra el carácter creativo de la mente humana, imagen de la divina, volviéndola creadora de un mundo. Esta línea se ha nutrido con estudios de obras como De coniecturis (1440-4) o De ludo globi (1463) que completan el cuadro de un mundo simbólico patrimonio exclusivo del arte humano del conjeturar.

Sin embargo, se ha puesto poco énfasis en señalar que el diálogo inmediatamente anterior en la serie: el segundo diálogo de Idiota. De sapientia. Este breve texto presenta una reflexión sobre el acto de concebir y el concepto, y postula la noción de concepto absoluto. Solo si se tiene en cuenta tal reflexión se ubica correctamente el tema de la subjetividad en el pensamiento cusano.

En De sapientia II el Cusano pone en juego una suerte de argumento ontológico. Declara que toda pregunta sobre Dios presupone lo preguntado. Así, quien pretenda hacer un concepto de Dios advertirá que Él es el concepto del concepto. Ambos, se fundan para el Cusano en el concepto del concepto que, por definición, debe ser él mismo inconceptuable. Dios como 
concepto absoluto es la fuente o raíz de toda conceptuación. El Idiota o ignorante, a la sazón la voz del mismo Cusano, lo expresa con estos términos: "En primer lugar decías que el concepto acerca del concepto es el concepto de Dios dado que Dios es el concepto de los conceptos conceptuables"(De sap. II (h V n. 34)

Una vez que se ha establecido que Dios es tal concepto absoluto, el orador, interlocutor del ignorante, pregunta:

Ahora bien ¿no es la mente la que concibe? - Ig: Sin la mente no hay concepto. - Or: por tanto, siendo el concebir propio de la mente, entonces concebir un concepto absoluto no es sino concebir el arte de la mente absoluta (De sap. II (h V n. 34). ${ }^{3}$

El Cusano apoya su argumentación en el doble significado del término latino "conceptus" que refiere tanto la operación de concebir cuanto el producto de tal operación. Solo una mente concibe o conceptúa. Y esto no es ajeno a una realidad absoluta pensada como mente: la mente divina o absoluta concibe un concepto absoluto que es su Razón o Verbo, forma ideal e igualdad de todas las cosas formables en el cual todo sea encuentra prioritariamente. ${ }^{4}$

No es una novedad en el pensamiento cristiano la reflexión sobre el Lógos o Verbo de Dios, tampoco el hecho de declarar que en él todo se encuentra de un modo seminal o primordial. Sin embargo, quiero destacar el hecho de que el modelo para comprenderlo sea para el Cusano en modo de concebir de la mente humana. Solo a partir de esto el Verbo puede ser llamado Concepto Absoluto como mente que se concibe a sí misma sin alteridad.

2 ORATOR: .....Primo loco aiebas conceptum de conceptu, cum deus sit conceptionum conceptus, esse de deo conceptum.

3 Ibídem: Nonne mens est quae concipit? IDIOTA: Sine mente non fit conceptus. ORATOR: Concipere igitur cum sit mentis, tunc concipere absolutum conceptum non est nisi artem absolutae mentis concipere.

4 IDIOTA: Prosequere, quia in via es. ORATOR: Sed ars absolutae mentis non est nisi forma omnium formabilium. Sic video, quomodo conceptus de conceptu non est nisi conceptus ideae divinae artis. Si verum dico, responde. IDIOTA: Immo optime. Nam absolutus conceptus aliud esse nequit quam idealis forma omnium, quae concipi possunt, quae est omnium formabilium aequalitas. (35) ORATOR: Hic conceptus, ut puto, dei verbum seu ratio dicitur. IDIOTA: Qualitercumque a doctis dicatur, in eo conceptu sunt omnia, sicut illa, quae sine ratione praevia non prodeunt in esse, dicimus in ratione prioriter exsistere. Omnia autem, quae esse conspicimus, rationem sui esse habent, ut sint modo quo sunt et non aliter. Qui igitur in simplicitatem absolutae rationis in se omnia prioriter complicantis intuetur profunda mente, hic facit conceptum de per se seu absoluto conceptu. Et hoc erat primum, quod praemisi. ORATOR: Satis de hoc. Nunc adice, quomodo conceptus absolutae praecisionis sit prae|cisior de deo conceptus. 
El Cusano no repite frecuentemente la expresión "conceptus absolutus" pero tampoco la abandona. Reaparece en De non aliud (1462) cuando establece que Dios es la definición que se define a sí misma y a todo. ${ }^{5} \mathrm{La}$ fórmula de De non aliud es una suerte de síntesis de las consecuencias de la concepción absoluta: constituirse a sí mismo y a todo. Prueba de la relevancia de este tópico en el escrito es el subtítulo con el que aparece en un manuscrito encontrado hacia finales del siglo XX: Eiusdem de non aliud ac etiam de deffinitione omnia diffinienti (Reinhardt, 1986). En las Propositiones que se posponen al texto vuelve a formularlo de manera sintética en términos de intelecto que se entiende a sí mismo:

Quien ve que Dios no es otro, ni de todo aquello que entiende, ni de todo aquello que es entendido, ese tal ve a Dios que da al intelecto aquello que es no-otro que intelecto que entiende, y para lo inteligible, aquello que es no-otro que inteligible por el intelecto, y que el intelecto que entiende no es otro del intelecto.(De non aliud, $\left(\mathrm{h} \mathrm{XIII,} \mathrm{n.124))^{6 }}\right.$

Cabe destacar además que toda la argumentación es sostenida en este texto por dos fuentes que se presentan al mismo tiempo como una suerte de autoridad: Dionisio, llamado máximo teólogo, y Proclo, diádoco de Platón. Al mismo tiempo, el Cusano señala contra la evidencia histórica que advendrá con posterioridad que Proclo sigue a Dionisio, estableciendo entre ellos una afinidad doctrinal innegable. Tal afinidad también es relativa al tema que nos ocupa.

En en escrito anterior y cercano a De non aliud, De Principio (1459) el Cusano presenta la noción de definición o concepto absoluto a través de un término leído en In Parmenidem de Proclo: “authypostaton”. Sin duda, al tomar contacto con esta noción procleana, el Cusano encontró algo que ya había pensado él mismo con anterioridad (D’Amico, 2021).

El uso del término "authypostaton" en griego procede de las versiones de Moerbeke que indica que su equivalente latino es "per se subsistens". El Cusano también sigue esta versión aunque prefiere mantenerlo en el grie-

\footnotetext{
5 De non aliud (h XIII, n. 4)NICOLAUS: ....quodsi toto nisu mentis aciem ad li non-aliud convertis, mecum ipsum definitionem se et omnia definientem videbis.

6 Decima nona: Qui videt, Deum non esse aliud nec ab omni eo, quod intelligit, nec ab omni eo, quod intelligitur, ille videt Deum dare intellectui, quod est non aliud quam intellectus intelligens, et intelligibili, quod est non aliud quam intelligibile ab intellectu, et quod intellectus intelligens non sit aliud ab intellecto.
} 
go original. El breve opúsculo, considerado por mucho tiempo un sermón, se abre con texto del evangelio de Juan: “¿Tu quién eres?”, se le pregunta a Jesús. Él responde: "Principium, qui et loquor vobis". ${ }^{7}$ Jesucristo se denomina a sí mismo "principio que habla". Lo propio del Principio es sin duda la indivisibilidad y la suficiencia (per se subsistens), sin embargo el término procleano “authypostaton", a diferencia de su traducción latina, señala un dinamismo que el Cusano quiere destacar. ${ }^{8}$ El Principio es uno e indivisible pero, al mismo tiempo, se autoconstituye. Proclo, pensador de lo uno, le ofrece una herramienta terminológica para destacar lo que el Cusano quiere: la unidad del principio solo puede ser entendida como unitrinidad, lo Uno puesto que se autoconstituye es Intelecto o Concepto. El Cusano afirma que si la unidad ha de considerarse Principio, debe necesariamente engendrar la razón de sí mismo, es decir su definición o lógos. $^{9}$ Trasladando esto a términos propios de la teología, el Principio es, en un sentido primario, el Padre que no puede constituirse como tal sino en el engendramiento del Hijo. Sin embargo, también el Hijo es Principio. Así, de un lado, no puede haber principio en la eternidad sin principiado en la eternidad; de otro, sólo puede verse lo principiado en la eternidad como principio principiado. ${ }^{10}$ Entre principio eterno y principio principiado eterno, debe haber un nexo de tal modo indisoluble que sea un principiado de ambos. Así pues, la esencia única de la eternidad puede ser concebida como principio - principio del principio - nexo entre ambos. Y, aunque más adelante, reconoce que el nombre "authypostaton" es inapropiado para este Principio --como lo es cualquier nombre-- afirma que si se acuerda en utilizarlo este nombre solo puede ser designar al principio único. ${ }^{11}$ En suma, para el Cusano la Trinidad no fue

\footnotetext{
7 Nicolás de Cusa sigue aquí el texto de la Vulgata cuya traducción es discutida.

8 De princ. (h X/2b) n.2: Primum igitur investigemus, si est principium. Plato, prout Proclus in commentariis Parmenidis scribit, asseruit hunc mundum ex seniori causa in esse prodiise, nam partibile non potest per se subsistere; quod enim per se subsistit, hoc est quod esse potest. Partibile autem, cum possit partiri, potest non esse. Unde cum, quantum est de se, possit partiri et non esse, patet quod non est per se subsistens sive authypostaton.

9 De princ.(h X/2b, n. 9): Et non possumus negare, quin se intelligat, cum melius sit se intelligente. Et ideo rationem sui seu diffinitionem seu logon de se generat.

10 De princ.(h X/2b, n.10): Et ne haesites filium esse principium, adverte principium esse aeternum, et quod omnia, quae videntur in aeternitate, sunt aeternitas. Tunc vides quod non potest esse principium in aeternitate sine principiato in aeternitate. Videre autem principiatum est principium principiatum.

11 Cf. De princ h X/2b n. 18-20.
} 
ajena a la tradición platónica. Platón y Proclo llamaron "Uno" a aquello que engendra la inteligencia de sí. ${ }^{12}$

\section{Mente divina, mente humana: intentio}

Si volvemos la mirada a la estructura de los diálogos del Idiota, advertimos pues que el tratamiento de la humana mens sigue al tratamiento de la divina mens concebida como aquella que engendra su propio concepto o definición. Si la mente humana es imagen de aquella su operación ha de ser semejante.

El término "mens" procede de la tradición agustiniana y el Cusano identifica lo que denomina así con lo que otras tradiciones, fundamentalmente la procedente de Colonia, denominan "intellectus". Mens e intellectus son equivalentes para el Cusano pero deben diferenciarse de anima. De hecho en el diálogo se aclara que "anima" es el nombre de la mens ex officio, esto es por su función en el cuerpo. "Mens", en cambio, señala la operación desligada del cuerpo. Solo como intelecto o mente el hombre puede ser llamado viva imago dei. Se trata del paralelismo entre dos mentes: una infinita, la otra imagen de lo infinito. ${ }^{13}$ Toda la fuerza de la argumentación está puesta en la vis creativa subrayada en este "viva". Precisamente, la mente divina y la humana son semejantes en esto: ambas crean pero mientras la divina mens posee una vis entificativa, crea entes, la humana mens posee una vis assimilativa, simboliza volviendo todo a suo modo.

El paralelismo que ya había sido trazado por el Cusano en De Coniecturis, vuelve a ser considerado en el tratado De Beryllo (1458). En esta obra, el Cusano sintetiza toda su filosofía en cuatro proposiciones y afirma que la primera de estas proposiciones contiene (complicat) el resto. Con esta advertencia cusana, sabemos que bastará que entendamos bien esta primera

\footnotetext{
12 De princ. (h $\mathrm{X} / 2 \mathrm{~b}, \mathrm{n} .11$ ): Et ita vides principium et principiatum principium et principiatum principii utriusque esse unam aeternitatis essentiam, quam Plato vocat unum.

13 De mente (h V n. 57) .... IDIOTA: Puto certe. Nam alia est mens in se subsistens, alia in corpore. Mens in se subsistens aut infinita est aut infiniti imago. Harum autem, quae sunt infiniti imago, cum non sint maximae et absolutae seu infinitae in se subsistentes, posse ali quas animare humanum corpus admitto, atque tunc ex officio easdem animas esse concedo. PHILOSOPHUS: Concedis igitur eandem esse mentem et hominis animam: mentem per se, animam ex officio?
} 
premisa para entender el sentido cabal de todo su pensamiento". Conviene entonces que la leemos completa:

Conviene que, en primer lugar, consideres que lo uno es el primer principio y se llama, según Anaxágoras, intelecto, del cual todo es llevado al ser a fin de que el mismo se manifieste. Pues el intelecto se complace en mostrar y comunicar la luz de su inteligencia. Por lo tanto el intelecto hacedor, porque se constituye fin de sus obras, para que su gloria se manifieste de manera evidente, crea las sustancias cognoscitivas, que pueden ver su verdad, y a ellas se brinda el mismo hacedor en modo tal que puedan captarlo como visible. Saber esto es lo primero; en ello todo lo que se ha de decir está contenido complicadamente.(De Beryllo (h XI/ 1 n. 4) (14 $^{14}$

La disyunción exclusiva entre henología o filosofía del espíritu desaparece: el primer principio es "Uno" e "Intelecto". El "Ser" es lo que procede del "Uno-Intelecto", es decir no es primero. La mención de Anaxágoras proviene de la lectura de la Metafísica de Aristóteles, quien afirma que postulando el nous como principio, Anaxágoras se habría comportado como un "sobrio entre borrachos". Otras fuentes implícitas se hacen explícitas en otros pasajes de la obra: el nous demiourgikós del Timeo de Platón o lo que se denomina conditor intellectus. Esta fórmula aparece reiteradamente en In Parmenidem de Proclo en la versión latina de Moerbeke. ${ }^{15}$

Lo Uno debe ser Intelecto no solo para autoconstituirse sino para comunicar ad extra. La indivisibilidad imparticipable de lo Uno absoluto engendra de sí su concepto ad intra y también sale de sí para participarse en su similitud ad extra. Pero además, el intelecto hacedor se hace visible para ser visto. ${ }^{16}$ El principio reclama ser inteligido para lo cual no solo debe ser él mismo inteligible sino que debe crear intelectos.

\footnotetext{
14 Oportet te primum attendere unum esse primum principium, et id nominatur secundum Anaxagoram intellectus, a quo omnia in esse prodeunt, ut se ipsum manifestet. Intellectus enim lucem suae intelligentiae delectatur ostendere et communicare. Conditor igitur intellectus, quia se finem facit suorum operum, ut scilicet gloria sua manifestetur, creat cognoscitivas substantias, quae veritatem ipsius videre possint, et illis se praebet ipse conditor modo quo capere possunt visibilem. Hoc scire est primum, in quo complicite omnia dicenda continentur $\gg$

15 Cf. In Parm.II (Co 733-735), cod.cus. 186 f. 26 v; III (Co 804 ff.) cod.cus. 186 fol. 41 v; 42 r. El Cusano volverá a mencionarla cuando conozca la Theología Platónica (In Plat. Theol. V 12).

16 De Beryllo (h XI/ 1 n. 26) : « Sicut si conditor intellectus foret visus volens suam virtutem videndi ostendere, omne visibile, in quo se ostendat, conciperet..."
} 
Lo que comunica el hacedor para que sea visto es denominado por el Cusano intentio y aquellos que pueden ver, son al mismo tiempo capaces de intentiones. Leemos:

Luego de esto, traigamos a la memoria lo que antes dije acerca de la intención es decir, cómo la creatura es intención del creador (intentio creatoris) y consideremos que la intención es la muy verdadera quididad suya. Pues según un simil, si cuando alguien nos habla alcanzamos la quididad de la elocución, no alcanzamos sino la intención del que habla (...) De este modo, lo sensible es como si fuera la palabra del hacedor, en la cual se contiene su intención, captada la cual sabemos la quididad y reposamos. Ahora bien, la causa de la intención es la manifestación; pues pretende así manifestarse quien habla, o sea el intelecto hacedor. Por lo tanto, aprehendida la intención, que es la quididad de la palabra, tenemos "lo que es ser". Pues "lo que es ser" en el intelecto es aprehendido en la intención, como en la casa concluida se aprehende la intención de quien ha edificado, la cual estaba en su intelecto(De Beryllo, (h $\mathrm{XI} / 1$, n. 54) $)^{17}$.

El paralelismo aparece nuevamente. En esta misma obra el hombre es llamado "mensura rerum" y "secundus deus": la mente humana (mens) mide (mensurat) todas las cosas y crea (creat) entes racionales y formas artificiales, que son sus intentiones.

Debemos aclarar correctamente el sentido del término "intentio". Si bien el Cusano muchas veces la asocia con "verbum" o "conceptus" no debe entenderse como parte de un proceso de conceptualización tal como aparece en algunos autores escolásticos. ${ }^{18} \mathrm{El}$ movimiento que presenta el Cusano es inverso: el movimiento intencional tiene siempre su punto de partida en el intelecto y no en la cosa. Y esa intención es inaprehensible para nosotros: no podemos conocer la quididad de la creatura así como tampoco podemos conocer a través de un discurso, la intención del hablante de un modo completo. La regla de la docta ignorantia a la que el Cusano no ha re-

\footnotetext{
${ }^{17}$ Post haec ad memoriam revocemus ea quae supra dixi de intentione, scilicet quomodo creatura est intentio conditoris, et consideremus intentionem esse verissimam quiditatem eius. Nam a simili, cum quis nobis loquitur, si nos quiditatem attingimus sermonis, non nisi intentionem loquentis attingimus [...) ut sensibile sit quasi verbum conditoris, in quo continetur ipsius intentio, qua apprehensa scimus quiditatem et quiescimus. Est autem intentionis causa manifestatio; intendit enim se sic manifestare ipse loquens seu conditor intellectus. Apprehensa igitur intentione, quae est quiditas verbi, habemus 'quod erat esse'. Nam 'quod erat esse' apud intellectum est in intentione apprehensum, sicut in perfecta domo est intentio aedificatoris apprehensa, quae erat apud eius intellectum.

18 Cf. por ejemplo, Thomas Aquinas, Summa contra Gentiles, I q 53 “... intellectus per speciem rei formatus intellegendo format in seipso quandam intentionem rei intellectae, quae est ratio ipsius, quam significat definitio".
} 
nunciado a lo largo de todo su pensamiento afirma que lo primero que el espíritu humano sabe es que la verdad es inaccesible en sí misma y que su mostración solo es posible en la alteridad, por esto es necesariamente parcial.

Así pues como hay un vínculo entre intelecto, comunicabilidad e intención, lo hay también entre comunicabilidad, intención y alteridad. Tal como se sintetiza en la fórmula cusana: "Intentio autem est similitudo intendentis, quae est communicabilis et receptibilis in alio". ${ }^{19}$

\section{El símil del lenguaje: Grammatica absoluta}

En las obras que hemos mencionado el símil del lenguaje ocupa un lugar muy destacado: Jesús dice de sí mismo no solo ser Palabra (Verbum, Lógos) sino Palabra que habla y su discurso es la creación. En un Sermón de Navidad pronunciado en Brixen en 1456, el Cusano ya había caracterizado la creación como locución o discurso divino. ${ }^{20}$ Tal discurso se da porque el Principio "quiere" ser conocido.

En la obra De aequalitate (1460) a propósito de una aclaración acerca de este "quiere" (vult) que había utilizado en De Beryllo, ${ }^{21}$ el Cusano vuelve a desarrollar una doctrina del alma humana (Flasch, 1998: 483). Esto es adecuado si pensamos que el espíritu humano es el gran intérprete de la creación. En esta obra, el Cusano opta por el término "anima" y la caracteriza con el vocabulario agustiniano de De Trinitate (Cranz, 1996). La recurrencia explícita a Agustín se explica en la medida en que el propósito del Cusano es hallar en la relación íntima del alma con su palabra interior (verbum interior) un modelo del engendramiento trinitario (Panaccio, 1999:118-119). Las relaciones entre memoria, entendimiento y voluntad hacen nacer el verbo interior así como el Padre hace nacer de sí su Palabra eterna. De esta manera, el alma encuentra todo en sí misma y ve todo más verdaderamente en sí misma que fuera de sí. Midiendo todo se mide a sí misma. ${ }^{22} \mathrm{Y}$ al hacerlo se descubre como límite nocional "El alma ve el límite en todos los limita-

19 De Beryllo (h XI/1 n. 37)

20 Sermo CCLVIII (h XIX/5 n. 2): Dicit autem ex alto incipiens: «Multivarie multisque modis» etc. Deus non loquitur nisi semel, et haec locutio in variis, per quos locutus est, varia est. Loqui est explicatio verbi mentalis. Creare Dei et loqui quoad Deum idem sunt .

21 De aequ. (h X/1, n. 3): Legisti in Beryllo nostro, quomodo intellectus vult cognosci... 
dos. Y dado que no hay límite del límite, ve que ella es el límite nocional no limitado, sin alteridad"'(De aequ. (h X/1, n. 10)). ${ }^{23}$

Puesto que el alma ve la noción del mundo en sí, ella es nombrada con todos los nombres. Su discurso interior es llamado verbo o palabra nocional de la totalidad o nombre de todo nombre. ${ }^{24}$

Así el concepto mental es identificado con la palabra interior y tal concepto mental complica o contiene los límites de todas las cosas, como en una especie de gramática absoluta. Leemos:

Así como si el intelecto fuese la gramática absoluta, el cual en su precisa razón o definición se conociera a sí mismo, también en ella conocería todo lo que pudiera ser sabido o bien extrínsecamente ser dicho o enunciado o dado a conocer por cuanto aquella razón abrazaría universal y particularmente todo aquello que pueda ser sabido $\mathrm{y}$ dicho de cualquier forma que fuere de modo que nada pudiese ser dicho gramaticalmente que no correspondiese ser dicho coincidente conforme a aquella razón y modalidad de dicción (De aequ. (h $\mathrm{X} / 1, \mathrm{n} .18)) .{ }^{25}$

El adjetivo "absoluta" no debe ser entendido como algo relativo al orden de lo divino sino más bien se refiere a una gramatica propia del espíritu humano en la cual todo se da sin diversidad ni alteridad, es decir de un modo desvinculado. Aquí, como en otras ocasiones, el Cusano recurre a la ejemplificación por las artes liberales. Las artes del trívium en este caso y su vinculación con el lenguaje resulta un buen "enigma" para comprender lo absoluto, no porque se trate de un contenido innato de la mente, sino porque estas artes están siendo en ella como posibilidad. Esto es extensible a todas

22 De aequ. (h X,/1 n. 9): Et ita habes, quomodo anima pergit per se ad omnia alia et nihil reperit in omni varietate intelligibile, nisi quod in se reperit, ut omnia sint sua similitudo. Et in se verius omnia videt quam sint in aliis ad extra. Et quanto plus egreditur ad alia, ut ipsa cognoscat, tanto plus in se ingreditur, ut se cognoscat. Et ita, dum per proprium intelligibile alia intelligibilia mensurare et attingere satagit, per alia intelligibilia sum proprium intelligibile sive seipsam mensurat

23 Anima videt terminum in omnibus terminatis. Et cum termini non sit terminus, videt se terminum notionalem interminum sine alteritate.

24 Ibídem: Anima, dum se videt, < videt $>$ intra se habere notionem mundi omnem omnium mundi notiones complicantem; videt in se esse verbum seu conceptum notionalem universorum et nomen omnium nominum, per quod de omni nomine notionem facit; et videt omnia nomina suum nomen explicare, cum nomina non sint nisi rerum notiones. Et hoc est, quod ipsa anima se videt omnibus nominibus nominari.

${ }^{25}$ Sicut si grammatica absoluta foret intellectus, qui se in sua praecisa ratione seu diffinitio ne cognosceret: in illa etiamomnia, quaesciriautextrinsecediciveleloquisiveproferripossent, cognosceret, quia ratio illa universaliter et particulariteromniataliaqualitercumquescibilia et dicibiliacomplecteretur, itaquod nihil dicipossetgrammatice, quinsecundumillamrationem et dicibilitatem cum illa rationecoincidentemdicioporteret. 
las disciplinas liberales, consideradas asimismo como diversos modos del discurso humano. Leemos en De ludo globi (1463) esta vez en relación con las artes del quadrivium:

El alma racional inventa disciplinas tales como la aritmética, la geometría, la música y la astronomía, y descubre que están dentro de su poder pues son descubiertas y puestas de manifiesto por los hombres. Y porque dichas disciplinas son incorruptibles y permanecen siempre sin cambio, el alma se observa a sí misma como algo incorruptible, cierta y siempre permanente.(De ludo globi $(\text { h.IX,n. 93) })^{26}$

El carácter innato de las artes liberales no es una originalidad cusana. Pensadores muy frecuentados por él como Agustín o Eriúgena así lo sostuvieron. La originalidad cusana consiste en que el carácter innato o connatural lo tiene la "complicación" de todo contenido pero de manera indeterminada y como posibilidad, en una suerte de estructura formal. Así como el concepto absoluto no contiene ideas eternas sino la posibilidad misma del ser, del mismo modo el concepto mental encierra la posibilidad de desplegarse a sí mismo en la configuración de sus artes. En el concepto mental como en el concepto absoluto se encuentra indistintamente y como intentio lo que habrá de ser comunicado en la alteridad como discurso: límite entitativo de todo en el caso de Dios, límite nocional de todo en el caso del hombre.

De este modo, el pensamiento cusano se ubica en un lugar de transición reasumiendo en sí las fuentes neoplatónicas que conoce y auspiciando un nuevo pensar desde una metafísica de la subjetividad absoluta y humana, ambas entendidas como el ámbito de la posibilidad que se despliega en actualizaciones sucesivas.

Recebido em 05/03/2021 e aprovado em 15/04/2021

\footnotetext{
26 Et invenit disciplinas, scilicet atrithmeticam, geometriam, musicalem et astronomicam, et illas in sua virtute complicari experitur. Sunt enim illae disciplinae per homines inventae et explicatae. Et cum sint incorruptibiles et semper eodem modo manentes, et vere videt anima se ipsam incorruptibilem semper vere permanentem..."
} 


\section{REFERENCIAS BIBLIOGRÁFICAS}

\section{Fuentes citadas}

NICOLAUS CUSANUS, Idiota. De sapientia, De mente, De staticis experimentis. Opera Omnia. Volumen V. Edición: R. Steiger-L. Baur, Hamburg: Felix Meiner, 1983.

. De deo unitrino principio, Tu quis es (De principio). Opera Omnia. Volumen X. Opuscula II.Fasciculus 2 b. Edición:. K. Bormann and A.D. Riemann, Hamburg: Felix Meiner, 1988.

. Directio Speculantis seu de non aliud, Opera Omnia. Volumen XIII. Edición: L. Baur- P. Wilpert. Hamburg: Felix Meiner, 1950.

. De Beryllo. Opera Omnia. Volumen

. De aequalitate. Opera Omnia. Volumen X, Opuscula II. Edición: H-G. Senger, Hamburg: Meiner, 2001.

. Dialogus de ludo globi. Opera Omnia. Volumen IX, Edición: H-G. Senger, Hamburg: Meiner, 1998.

. Sermones IV. Opera Omnia. Volumen XIX. Edición: H.D. Riemann, H. Schwaetzer und F-B Stammkötter. Hamburg: Felix Meiner, 2005.

PROCLUS, Commentaire sur le Parménide de Platon. Traduction de Guillaume de Moerbeke. Tome II: Livres V à VII et Notes marginales de Nicolas de Cues. Édition critique par Carlos Steel. Suivi de l'édition des extracts du Commentaire sur le Timée, traduits par Moerbeke, Leuven-Leiden: Leuven University Press- Brill, 1985.

\section{Bibliografía secundaria citada.}

BEIERWALTES, W. Platonismus und Idealismus. Frankfurt am Main: Klostermann, 1972.

. Identität und Differenz. Zum Prinzip cusanischen Denkens, Opladen: Westdeutscher Verlag, 1980.

. "The Legacy of Neoplatonism in F. W. J. Schelling's Thought". International Journal of Philosophical Studies, 10: 4, 2002, 393428.

BENZ, Huber. Individualität und Subjektivität. Interpretationstendenzen in der Cusanus-Forschung und das Selbstverständnis des Nikolaus von Kues. Münster: Aschendorff, 1999. 
CRANZ, E. "The De aequalitate and De principio of Nicholas of Cusa". In: G. Christianson- Th. M. Izbicki (eds.), Nicholas of Cusa on Christ and Church. Leiden: Brill, 1996, 271-280.

D’AMICO, C. "La recepción de la noción procleana de authypóstaton en Nicolás de Cusa". Revista Latinoamericana de Filosofía, 47:1, 2021 (en prensa)

FLASCH, Kurt. Nikolaus von Kues. Geschichte einer Entwicklung. Vorlesungen zur Einführung in seine Philosophie, Frankfurt am Main: Vittorio Klostermann, 1998.

PANACCIO, C. Le discours intérieur de Platon à Guillaume d'Ockham, París: Seuil, 1999.

REINHARDT, K. "Eine bisher unbekannte Handschrift mit Werken des Nikolaus von Kues in der Kapitelsbibliothek von Toledo". Mitteilungen und Forgungsbeiträge der Cusanus-Gesellschaft, 17, 1986, 96-141.

STANFIELD, Ph. Hegel. The Consummate Neoplatonist. Tesis de doctorado inédita, año 2017. Disponible en academia.edu (ex. 23 de marzo 2021. https://www.academia.edu/35220519/Hegel the consummate Neoplatonist_B_pdf).

STROK, N. "Praecisio veritatis inattingibilis: La presencia de Nicol'ás de Cusa en la historiografía filosófica moderna a la luz de Gründrisse der Geschichte der Philosophie de W. G Tennemann”. En H.J. Gonzalez Ríos (ed.), La dimensión simbólica del pensamiento de Nicolás de Cusa. Genealogía y proyección, Buenos Aires, Círculo de Estudios Cusanos de Buenos Aires, 2015, 347-363.

ZEYER, K. Cusanus in Marburg. Hermann Cohens und Ernst Cassirers produktive Form der Philosophiegeschichtsaneignung. Münster: Aschendorff, 2015. 